\title{
Nanosized Polyanilines Prepared with Ultrasound Oxidative Polymerization at Different Frequencies
}

\author{
Kazuki Fukui ${ }^{1}$, Erasto Armando Zaragoza-Contreras ${ }^{1,2}$, \\ Kohsuke Hirano ${ }^{1}$ and Takaomi Kobayashi ${ }^{1, *}$ \\ 'Department of Materials Science and Technology, Nagaoka University of Technology, \\ Kamitomioka 1603-1, Nagaoka Niigata 940-2188, Japan \\ ${ }^{2}$ Department of Chemical Engineering and Materials, Centro de Investigación en Materiales \\ Avanzados, S.C. Miguel de Cervantes No. 120, Chihuahua, Chih., México
}

(Received February 16, 2015; accepted September 4, 2015)

Key words: conductivity polymer, polyaniline, ultrasound, nanoparticle

Polyaniline (PANi) nanoparticles were successfully prepared by applying ultrasound (US) irradiation during the oxidative polymerization of aniline in water. The US frequencies applied were 23, 43, and $141 \mathrm{kHz}$ with varied exposure times. The formation of PANi nanoparticles was confirmed using laser diffraction particle size analysis (LDPA), scanning electron microscopy (SEM) and transmission electron microscopy (TEM). The results of LDPA revealed that, when the US exposure lasted from 10 to $120 \mathrm{~min}$, the nanosization of PANi was reduced at $141 \mathrm{kHz}$, whereas at a lower US frequency PANi formed aggregates with a larger size. The results of TEM indicated that the US frequency affected the aggregate size and particle size, meaning that a higher US frequency dispersed more efficiently nanosized particles.

\section{Introduction}

Conducting polymers have been extensively developed and expanded into industrial applications rapidly since their discovery by Shirakawa et al. in the late 1970s.(1) They developed the metal-like conductivity of polyacetylene after doping a polymer with iodine vapor and also investigated new conducting polymer systems. Nowadays, polypyrrole (PPy), polyaniline (PANi), polythiophene (PTs) and derivatives of their copolymers form the most extensive class of conductive polymers, and their uses in a variety of applications have been widely studied. ${ }^{(2-7)}$ The conductivity of these polymers results from mobile charge carriers introduced into conjugated $\pi$-systems through the doping process. ${ }^{(8-10)}$

*Corresponding author: e-mail: takaomi@nagaokaut.ac.jp 
PANi (Fig. 1) is the most extensively studied conducting polymer, because it is highly conductive and stable at room temperature, and has a wide potential range when doped with protic acids. ${ }^{(11,12)}$ PANi was used in various applications, for example, anticorrosion coatings, ${ }^{(13-15)}$ photovoltaics, ${ }^{(16-18)}$ supercapacitors, ${ }^{(19-21)}$ and lithium secondary batteries. ${ }^{(22-24)}$ In the case of preparation, the synthesis method for PANi is well established and several methods, such as electrochemical polymerization and chemical polymerization, have been used for it. ${ }^{(25-27)}$ Therefore, the preparation of nanosized PANi has become an attractive area of study and papers concerning nanosized PANi have appeared..$^{28-30)}$

In this work, we have developed a simple and effective chemical method of polymerization for the preparation of nanosized PANi using ultrasound (US) as a parameter to control aggregate size and particle size and geometry. The preparation and properties of nanosized PANi using US exposure are described, and the effect of US frequency is also discussed.

\section{Materials and Methods}

\subsection{Materials}

Aniline (Nacalai Tesque Inc., Japan) was used as the monomer, ammonium peroxodisulfate (APS) (Nacalai Tesque Inc., Japan) as the oxidizing agent, and trifluoroacetic acid (TFA) (Tokyo Chemical Industry Co., Ltd., Japan) as the doping agent. All reagents were used as delivered.

\subsection{Preparation of nanosized PANi using US}

Figure 2 shows the schematic procedure for the preparation of PANi nanosized particles. The PANi nanosized particles were polymerized as follows: aniline was dispersed in distilled water at of $0.01 \mathrm{M}$ and TFA was dissolved in distilled water at 0.01
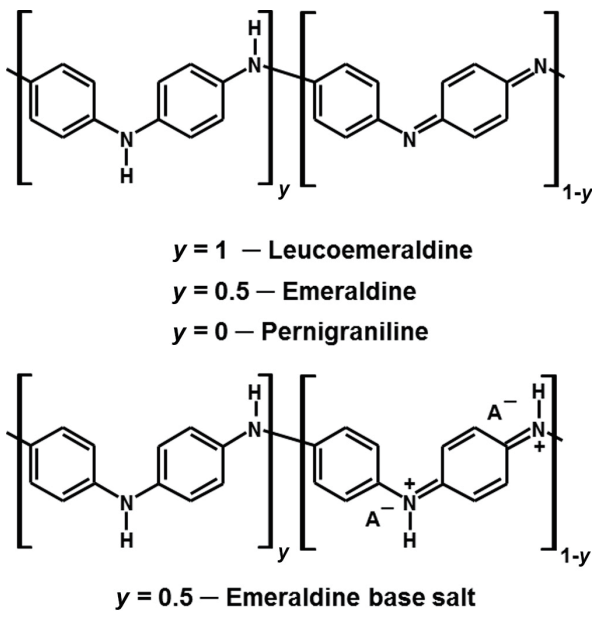

Fig. 1. Structure and oxidation states of PANi. 


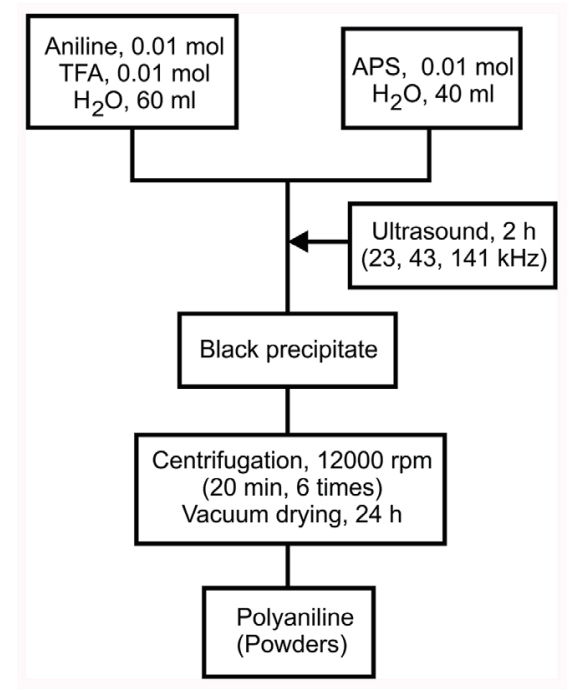

Fig. 2. Schematic procedure for the preparation of PANi by US oxidative polymerization.

M. Then, $40 \mathrm{ml}$ of the aqueous solution of aniline was mixed with $20 \mathrm{ml}$ of the aqueous solution of TFA. Then, the solution of aniline and TFA was added to $40 \mathrm{ml}$ of the 0.01 M APS aqueous solution used as the oxidizing agent. The polymerization then ensued. Subsequently, the clear solution was irradiated with US at a power of $50 \mathrm{~W}$ at each US frequency $(23,43$, or $141 \mathrm{kHz})$ with exposure times from 10 to $120 \mathrm{~min}$. The US power was maintained at $50 \mathrm{~W}$ using a power amplifier (HSA4012, NF, Japan). Finally, the reaction mixture was magnetically stirred for $22 \mathrm{~h}$. The black precipitate was washed with water and then acetone. The PANi particles were dried under vacuum for $24 \mathrm{~h}$.

\subsection{US equipment for oxidative polymerization}

Figure 3 show a scheme of the equipment used for the synthesis of PANi in the presence of US. The system was similar to that used in our previous work involving US exposure. ${ }^{(31-33)}$ The sample containing the monomer and TFA were set in a water bath $(8.5$ $\times 13.5 \times 13 \mathrm{~cm}^{3}$ ) with a 3-cm-diameter transducer on its sidewall. The samples were irradiated with US at various frequencies. The frequency was controlled by a function synthesizer (WF1943B, NF, Japan) and the power by a power amplifier (HSA4012, NF, Japan) as shown in Fig. 3. The water in the US system was maintained at $28{ }^{\circ} \mathrm{C}$ by a thermostated bath. The US power entering the system was determined by substituting the value of $\Delta T / \Delta t$ into $D_{\mathrm{p}}=m C_{\mathrm{p}}(\Delta T / \Delta t)$. Here, $C_{\mathrm{p}}$ is the heat capacity of water (4200 $\mathrm{J}$ $\left.\mathrm{kg}^{-1} \mathrm{~K}^{-1}\right)$ and $m$ represents the mass of the solvent $(\mathrm{kg})$. The powers dissipated in the US water bath were $8.5,9.0,8.7$, and $8.8 \mathrm{~W}$ for respective power values of $50 \mathrm{~W}$ for 23,43 , and $141 \mathrm{kHz}$. 


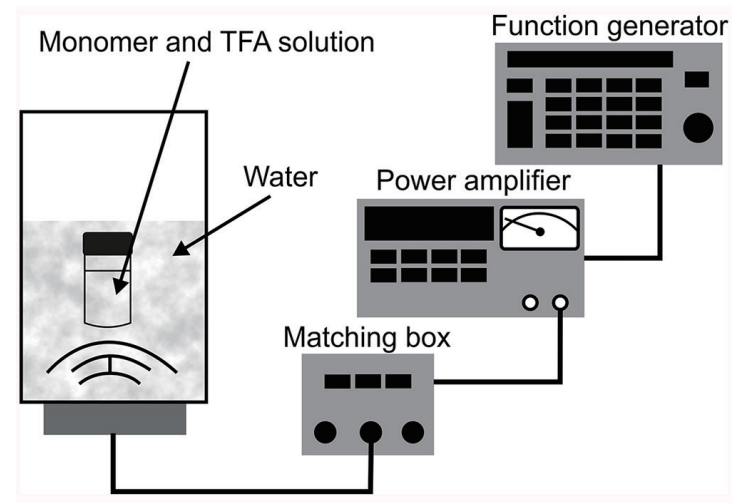

Fig. 3. Experimental setup for the US oxidative polymerization of aniline.

\subsection{Characterization}

The resultant PANi powders were characterized using an infrared spectroscope (Prestige-21; Shimadzu Corp., Japan) in the transmittance mode. The transmission spectra were obtained from compressed KBr-PANi pellets. The resolution of the spectra was 4 $\mathrm{cm}^{-1}$ for each spectrum. Additionally, a UV-VIS-NIR spectrophotometer (V-570; JASCO Corp., Japan) was used to characterize absorbance. For the UV-vis spectra, the PANi powders were dissolved in $N$-methyl-2-pyrrolidone at a concentration of $0.02 \mathrm{~g} / \mathrm{l}$ and then recorded. To evaluate the powder size distribution, laser diffraction particle size analysis (SALD-7000; Shimadzu Corp., Japan) (LDPA) was performed. The aggregate size and morphology of each polymer sample were observed by scanning electron microscopy (SEM, JSM-5300 LV; JEOL, Japan); before analysis, the samples were gold-coated using a quick-cool coater (Sanyu Denshi K.K., Japan). Particle size and morphology were characterized by field emission scanning electron microscopy (JSM-7401F, JEOL Ltd., Japan) for samples prepared by placing a droplet of the polymerization product on a holey-carbon-copper grid and then left to dry under laboratory conditions.

\section{Results and Discussion}

\subsection{Characterization of PANis formed at different US frequencies}

Figure 4 shows the effect of US irradiation time on the average aggregate size of PANi. In the polymerization experiments, the irradiation time was varied between 10 and $120 \mathrm{~min}$ at different frequencies and a power of $50 \mathrm{~W}$. For all irradiated PANi samples, the average aggregate size shifted similarly as the irradiation time was increased. The average aggregate size was about $15-18 \mu \mathrm{m}$ at $20 \mathrm{~min}$. When the US was on, during the polymerization period of $10 \mathrm{~min}$, the color solution changed to deep green, evidencing aniline oxidative polymerization. The PANi dispersion was sampled 


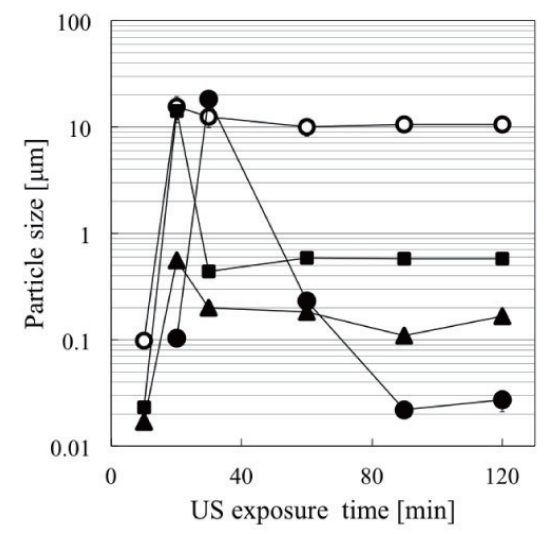

Fig. 4. Relationship between US irradiation time and average aggregate size of the PANi.

and analyzed every $10 \mathrm{~min}$. Each irradiated sample at a different frequency had an average aggregate distribution of less than $100 \mathrm{~nm}$ at $10 \mathrm{~min}$. However, the aggregate size at each frequency increased for irradiation times up to $20 \mathrm{~min}$. Then, at a longer period, the aggregate size decreased to $0.4,0.15$, and $0.02 \mu \mathrm{m}$ for frequencies at 23,43 , and $141 \mathrm{kHz}$, respectively. Figure 5 shows aggregate size distributions: a) without US irradiation and after 90 min of US irradiation at b) 23, c) 43, and d) $141 \mathrm{kHz}$. As seen, the $141 \mathrm{kHz}$ irradiation was effective for producing nanosized PANi aggregates.

The morphology of the PANi particles was observed by SEM (-S) and TEM (-T), and is shown in Fig. 6. The samples a-S and a-T correspond to PANi produced without US exposure for SEM and TEM images, respectively. The pictures show that the diameter of the PANi aggregate was about $10 \mu \mathrm{m}$. In contrast, US exposure at 23 (b-S), 43 (c-S) and $141 \mathrm{kHz}(\mathrm{d}-\mathrm{S})$ yielded SEM images revealing that US gave rise to more dispersed aggregates relative to those of (a-S). PANi particle morphology with magnetic stirring was primarily semispherical (a-T), although elongated particles were observed with nanometric sizes as shown in the inset. For polyanilines obtained in the presence of US, an evolution of the particle geometry results from the increased intensity of US irradiation; furthermore, most particles seem to be in the nanoscale as shown in the insets. As seen, at $23 \mathrm{kHz}(\mathrm{b}-\mathrm{T})$, the particles seem to evolve from the spherical shape, observed without US, to the fibrillar shape. Furthermore, at $43 \mathrm{kHz}$ (c-T), most of the particles exhibit a fibrillar shape, with a length of around $200 \mathrm{~nm}$. Finally, at $128 \mathrm{kHz}$ (d-T), the particles are smaller than the previous ones, with diameters below $50 \mathrm{~nm}$ and preserving the fibrillar geometry, suggesting that, with increasing US irradiation intensity, particle growth restriction occurs. The literature indicates that the use of US during PANi synthesis is important for controling the microstructure and hence properties of the polymer. ${ }^{(34,35)}$ Liu et al. performed aniline polymerization in the presence of US 


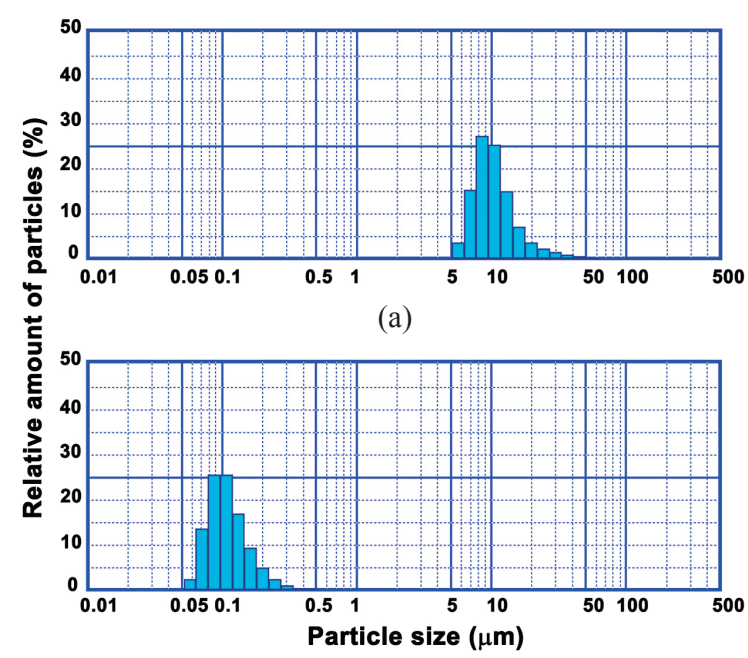

(c)

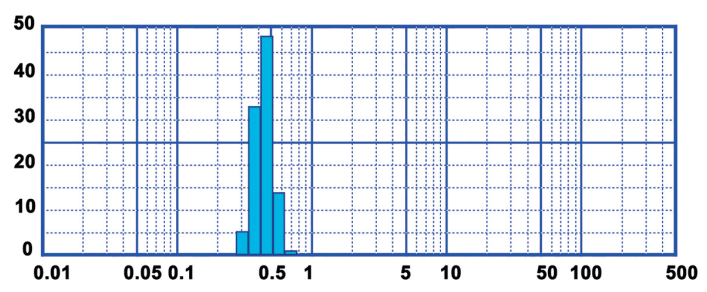

(b)

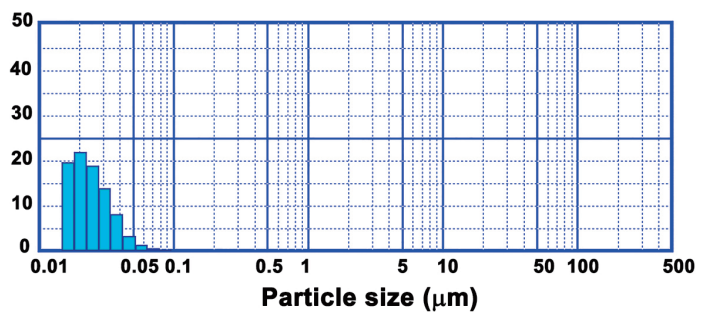

(d)

Fig. 5. (Color online) Aggregate size distributions of PANi determined by LDPA: synthesis (a) without US and at US frequencies of (b) 23, (c) 43, and (d) $141 \mathrm{kHz}$ for 90 min of irradiation.

(a)

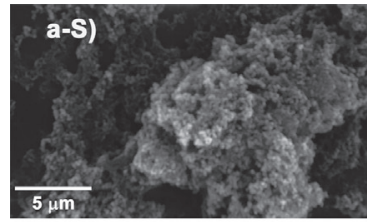

(b)

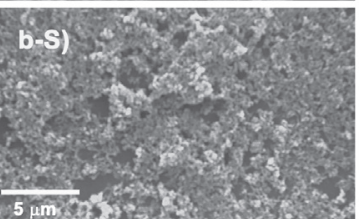

(c)
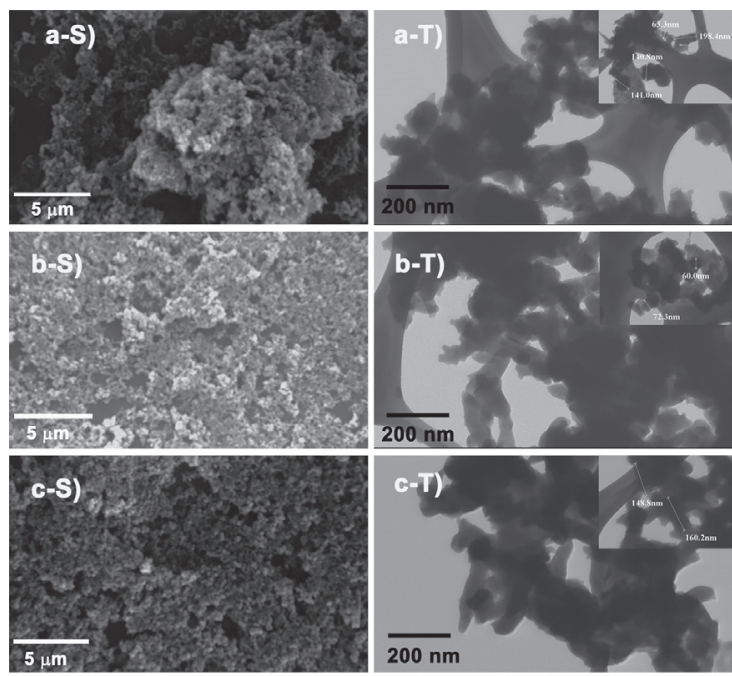

(d)

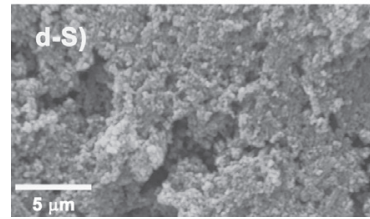

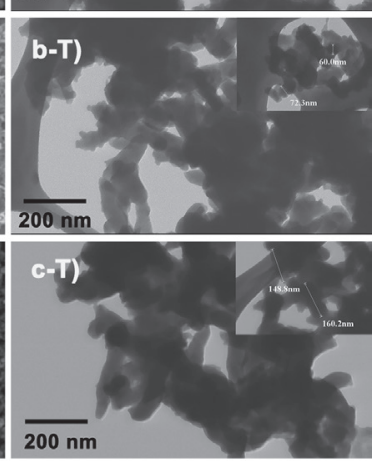

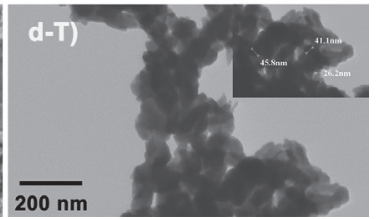

Fig. 6. SEM and TEM images of PANi: synthesized (a) without US and at US frequencies of (b) 23, (c) 43, and (d) $141 \mathrm{kHz}$. -S indicates SEM and -T indicates TEM. 
irradiation. They observed that most particles were thin hexagonal plates with diameters between 250 and $300 \mathrm{~nm}$ with a crystalline structure. Examples of extreme conditions generated by bubble collapse are the enhanced formation of free radicals, mechanical shocks, high shear gradients, and very rapid and efficient mixing in multiphase systems, which were some of the dealing forces for such a geometry. ${ }^{(36)}$ Husin et al. reported the synthesis of PANi under US irradiation at frequencies from 5 to $20 \mathrm{kHz}$. They obtained interpenetrated networks of short PANi fibers; additionally, they found that the frequency of the ultrasonic wave greatly affected the morphology of the PANi fiber; that is, the higher the ultrasonic wave frequency used, the smaller the diameter of PANi fibers. (37) Jing et al. observed, during PANi synthesis in the presence of sonication, that after oxidant agent addition and $4 \mathrm{~h}$ of polymerization, PANi nanofibers were still observed, indicating the positive effect of sonication in preventing the growth of primary nanofibers into thick fibers and especially the agglomeration of thick fibers into irregular particles. They suggested that primary PANi nanofibers, although their formation mechanisms are unclear, will grow into thick fibers with uneven surfaces and then aggregated to form irregular particles with magnetic stirring, whereas for US irradiation, the growth and agglomeration will be effectively hindered; thus, the morphology will be preserved in the final product. ${ }^{(38)}$ As shown in the literature, US generates a wide range of phenomena that can alter the structure and properties of PANi synthesized via oxidative polymerization. Many of these phenomena are still unclear; some of them may affect the polymerization mechanism, electrical properties or morphology, as seen above. The cavitation effect seems a decisive condition that allows control of the nanoparticle shape, as previously noted; however, more experiments are necessary to propose a model that clearly details the conditions that determine the geometry.

\subsection{Spectroscopic analyses of nanosized PANi}

To characterize the chemical structure in PANi, Fourier transform infrared (FTIR) spectra of samples synthesized at different US frequencies were recorded and are shown in Fig. 7. As seen, all samples exhibit similar peaks at 1137, 1303, 1470, and $1569 \mathrm{~cm}^{-1}$. The first peak at $1569 \mathrm{~cm}^{-1}$ corresponds to the stretching vibration of $\mathrm{C}=\mathrm{N}$ bonds in the quinoid ring. The peak at $1470 \mathrm{~cm}^{-1}$ is due to the $\mathrm{C}-\mathrm{H}$ stretching vibration of the benzenoid ring. The peaks at 1303 and $1237 \mathrm{~cm}^{-1}$ were assigned to the $\mathrm{C}-\mathrm{N}$ stretching vibration of the benzenoid ring. The peak at $1135 \mathrm{~cm}^{-1}$ was ascribed to the $\mathrm{C}-\mathrm{H}$ stretching vibration of the quinoid ring. Finally, the bands at 878 and $800 \mathrm{~cm}^{-1}$ were attributed to the $\mathrm{C}-\mathrm{H}$ bending of the aromatic ring of the benzenoid structure. The described bands coincide with the common signals reported in the literature for polyaniline in the emeraldine base form. ${ }^{(39,40)}$

It is known that PANi in fully reduced form has the chemical structure of leucoemeraldine (LB), in partially reduced form, of emeraldine (EB), and in fully oxidized form, of pernigraniline (PB) (Fig. 1)..$^{(41-43)}$ Therefore, the physical properties of PANi are strongly affected by the chemical structure of the polymer.

The UV-vis spectroscopy analysis of PANi samples dissolved in $\mathrm{N}$-methyl-2pyrrolidone was achieved. Figure 8 shows the absorption spectra of the PANi samples synthesized under different US irradiation intensities. The characteristic absorption 


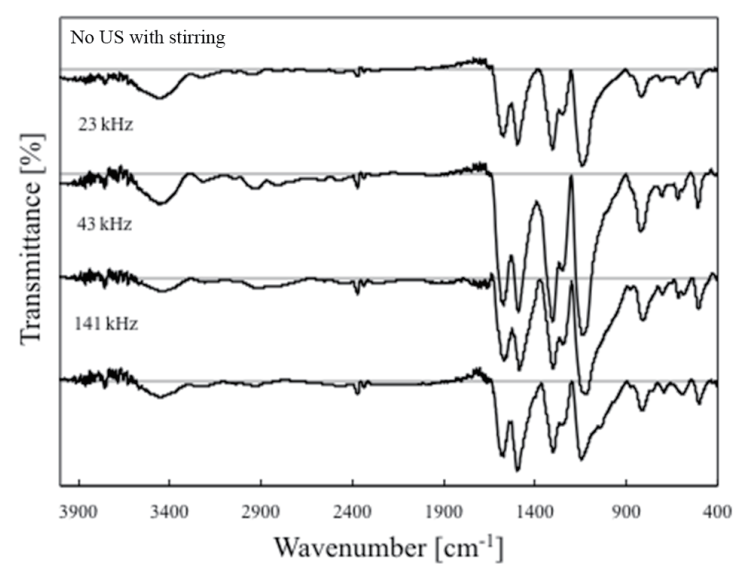

Fig. 7. FT-IR spectra of PANi produced without US and at US frequencies of 23, 43, and 141 kHz.

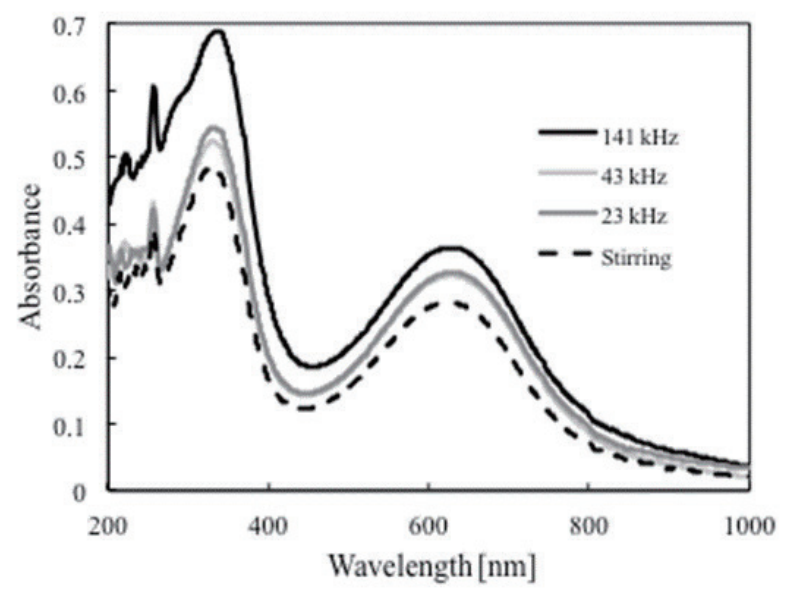

Fig. 8. UV-vis absorption spectra of solutions of PANi produced without US and at US frequencies of 23,43 , and $141 \mathrm{kHz}$.

bands of the PANi samples revealed broad peaks at around 648 and $320 \mathrm{~nm}$ owing to the $\pi-\pi^{*}$ transition of the benzenoid ring and the excitation absorption of the quinoid ring, respectively. ${ }^{(44,45)}$ The spectra implied the presence of both oxidized and reduced forms of PANi evidencing the EB form (Fig. 1).

Another feature to consider is the increased UV-vis absorption observed as a function of the ultrasonic intensity applied during the synthesis, regarding the polyaniline obtained without US. As for the morphology, the effects caused by US also impacted PANi optical properties in solution. In this regard, Chowdhury et al. reported that the 
absorption of a PANi colloid solution prepared with US (and in solution cast films) was higher at longer wavelengths than that of the colloidal solution prepared without US. They suggested that the difference in absorption at longer wavelengths may arise from the dissimilar electrical properties between the colloids prepared in the absence and presence of US. ${ }^{(46)}$ Xia and Wang compared the UV-vis absorption spectra of diluted colloid dispersions of PANi particles obtained in the presence and absence of US. They observed a lower absorbance at 860 and $300 \mathrm{~nm}$ for the sample obtained without US, indicating a lower doping level; that is, ultrasonic cavitation promoted the diffusion of acid molecules into the PANi chain. ${ }^{(47)}$ From our results, we agree with the reported literature since a variety of possible effects generated by the ultrasonic radiation can affect the optical behavior in the produced polymer solution. These effects are the result of, for example, the incidence of high shear rates, which affects polymerization variables, such as temperature and pressure, which in turn affect other variables, such as free radical production and doping agent diffusion, forming a chain of factors yet to be investigated.

\section{Conclusions}

PANi nanoparticles were successfully synthesized using oxidative polymerization in the presence of US irradiation. The effect of US frequency on the structure of PANi particles was studied. The PANi nanoparticles prepared at $141 \mathrm{kHz}$ exhibited the smallest particle size with the smallest aggregate size, indicating that a high US frequency is more effective to control aggregate and particle sizes, and also exerted a strong effect on the particle morphology.

\section{Acknowledgements}

This work was partially supported by the "Tri-Institutional Collaborative/Cooperative Educational Reform Project" of Nagaoka University of Technology for facilities used in support of this research. We also wish to acknowledge Laboratorio Nacional de Nanotechnología (CIMAV) and Wilber Antunez and Claudia Hernández for their collaboration during this research.

\section{References}

1 H. Shirakawa, E. J. Louis, A. G. MacDiarmid, C. K. Chiang and A. J. Heeger: J. Chem. Soc. Chem. Comm. 16 (1977) 578.

2 S.-Y. Hong, Y. M. Jung, S. Bin Kim and S.-M. Park: J. Phys. Chem. B 109 (2007) 3844.

3 A. Moliton and R. C. Hiorns: Polym. Int. 53 (2004) 1397.

4 J. Jang: Adv. Polym. Sci. 199 (2006) 189.

5 R. Waltman and J. Bargon: Can. J. Chem. 64 (1986) 76.

6 U. Lange, N. V Roznyatovskaya and V. M. Mirsky: Anal. Chim. Acta 614 (2008) 1.

7 M. Gerard, A. Chaubey and B. D. Malhotra: Biosens. Bioelectron. 17 (2002) 345.

8 J. L. Brédas, B. Thémans and J. M. André: Phys. Rev. B 27 (1983) 7827.

9 A. O. Patil, A. J. Heeger and F. Wudl: Chem. Rev. 88 (1988) 183.

10 J. Bakalis, A. R. Cook, S. Asaoka, M. Forster, U. Scherf and J. R. Miller: J. Phys. Chem. C 118 (2014) 114. 
11 W. Łużny and E. Bánka: Macromolecules 33 (2000) 425.

12 A. G. MacDiarmid, J. C. Chiang, A. F. Richter and A. J. Epstein: Synth. Met. 18 (1987) 285.

13 S. Sathiyanarayanan, S. S. Azim and G. Venkatachari: Synth. Met. 157 (2007) 751.

14 S. Radhakrishnan, C. R. Siju, D. Mahanta, S. Patil and G. Madras: Electrochim. Acta 54 (2009) 1249.

15 K. G. Conroy and C. B. Breslin: Electrochim. Acta 48 (2003) 721.

16 H. Bejbouji, L. Vignau, J. L. Miane, M.-T. Dang, E. M. Oualim, M. Harmouchi and A. Mouhsen: Sol. Energ. Mat. Sol. C. 94 (2010) 176.

17 H. Ashassi-sorkhabi, E. Asghari and A. Mirmohseni: Iran. Polym. J. 17 (2008) 711.

18 M. Ibrahim, M. Bassil, U. B. Demirci, T. Khoury, G. El Haj Moussa, M. El Tahchi and P. Miele: Mat. Chem. Phys. 133 (2012) 1040.

19 K. Zhang, L. L. Zhang, X. S. Zhao and J. Wu: Chem. Mat. 22 (2010) 1392.

20 Q. Wu, Y. Xu, Z. Yao, A. Liu and G. Shi: ACS Nano 4 (2010) 1963.

21 Z.-F. Li, H. Zhang, Q. Liu, L. Sun, L. Stanciu and J. Xie: ACS Appl. Mater. Interfaces 5 (2013) 2685.

22 J. Manuel, P. Raghavan, C. Shin, M.-Y. Heo, J.-H. Ahn, J.-P. Noh, G.-B. Cho, H.-S. Ryu and H.-J. Ahn: Mater. Res. Bull. 45 (2010) 265.

23 K. S. Ryu, K. M. Kim, Y.-S. Hong, Y. J. Park and S. H. Chang: Bull. Korean Chem. Soc. 23 (2002) 1144.

24 K. S. Ryu, S. K. Jeong, J. Joo and K. M. Kim: J. Phys. Chem. B 111 (2007) 731.

25 M. C. Miras, D. F. Acevedo, N. Monge, E. Frontera, C. R. Rivarola and C. A. Barbero: Open Macromol. J. 1 (2008) 58.

26 E. T. Kang, K. G. Neoh and K. L. Tan: Prog. Polym. Sci. 23 (1998) 277.

27 G. Ćirić-Marjanović: Synth. Met. 177 (2013) 1.

28 D. Li and R. B. Kaner: J. Am. Chem. Soc. 128 (2006) 968.

29 L. I. Dan, J. Huang and R. B. Kaner: Accounts Chem. Res. 42 (2009) 135.

30 J. Stejskal, I. Sapurina and M. Trchová: Prog. Polym. Sci. 35 (2010) 1420.

31 J. A. Venegas-Sanchez, M. Tagaya and T. Kobayashi: Polym. J. 45 (2013) 1224.

32 J. A. Venegas-Sanchez, T. Motohiro and K. Takaomi: Ultrason. Sonochem. 20 (2013) 1081.

33 J. A. Venegas-Sánchez, M. Tagaya and T. Kobayashi: Ultrason. Sonochem. 21 (2014) 295.

34 Z. Mo, H. Shi, H. Chen, G. Niu, Z. Zhao and Y. Wu: J. Appl. Polym. Sci. 112 (2009) 573.

35 M. Atobe, A. N. Chowdhury, T. Fuchigami and T. Nonaka: Ultrason. Sonochem. 10 (2003) 77.

36 H. Liu, X. B. Hu, J. Y. Wang and R. I. Boughton: Macromolecules 35 (2002) 9414.

37 M. R. Husin, A. Arsad, A. Hassan and O. Hassan: Appl. Mech. Mater. 618 (2014) 50.

38 X. Jing, Y. Wang, D. Wu, L. She and Y. Guo: J. Polym. Sci. Polym. Chem. 44 (2006) 1014.

39 A. B. Samui, A. S. Patankar, R. S. Satpute and P. C. Deb: Synth. Met. 125 (2002) 423.

40 J. Yang, Y. Ding, G. Chen and C. Li: Eur. Polym. J. 43 (2007) 3337.

41 S. Palaniappan and A. John: Prog. Polym. Sci. 33 (2008) 732.

42 R. Ansari and M. B. Keivani: E-J. Chem. 3 (2006) 202.

43 M. Angelopoulos, G. E. Asturias, S. P. Ermer, A. Ray, M. E. Scherr, A. G. Macdiarmid, M. Akhtar, Z. Kiss and A. J. Epstein: Mol. Cryst. Liq. Cryst. 160 (1988) 151.

44 A. Dan and P. K. Sengupta: J. Appl. Polym. Sci. 106 (2007) 2675.

45 L. C. Mendes, A. P. S. Falco, M. S. Pinho and P. O. Marques: Mater. Res. 14 (2011) 466.

46 A. N. Chowdhury, M. Atobe and T. Nonaka: Ultrason. Sonochem. 11 (2004) 77.

47 H. Xia and Q. Wang: J. Nanopart. Res. 3 (2001) 401. 